\title{
INTERNATIONAL CLASSIFICATION OF SPINAL INJURIES
}

\author{
By Robert RoaF \\ University of Liverpool
}

AN ideal classification of spinal injuries, indeed, an ideal classification of any deformity, should include the following features:

(a) The aetiological factors.

(b) The mechanism or means through which the aetiological factors work.

(c) The salient anatomical and pathological features of the resultant lesion.

(d) An indication of the means by which restoration of normal alignment and stability can be obtained with greatest ease and safety.

While the empirical treatment of spinal injuries has reached a high standard of excellence the present descriptive classification of spinal injuries which commonly features in textbooks and journals fails to achieve the above ideals and is often ambiguous, inconsistent and misleading.

Four obvious examples of the shortcomings of the present classification are:

I. All injuries of the cervical spine in which the patient has facial or frontal injuries are usually termed 'hyperextension' injuries, yet such facial or frontal injuries can produce a wide variety of different anatomical lesions which require different modes of management. There are at least four quite different so-called hyperextension injuries ( $\mathrm{K}, \mathrm{L}, \mathrm{M}, \mathrm{N}$, fig. I). Incidentally, hyperextension is in many ways an unfortunate word as extension literally means increase in length or longitudinal distraction. The obstetrical term deflexion although ugly would be more accurate.

2. Many cervical spine lesions are attributed to flexion, yet it is obvious to every medical student that unless the cervical spine is previously pathologically stiff, hyperflexion cannot occur unless either the mandible or manubrium sterni are broken, as normally one's mandible impinges on the manubrium sterni before the limit of cervical flexion is reached.

3. Similarly, in the upper thoracic spine, hyperflexion can only occur if there are multiple fractures of the ribs and/or sternum.

4. Experiments on isolated vertebrae show that so-called 'hyperflexion' produces compression of the vertebral bodies and/or discs, not tearing of the interspinous ligaments: in other words, most so-called hyperflexion injuries are really examples either of vertical compression, or rotation, or lateral flexion, or of forward displacement, or distraction. Many are due to completely different mechanisms, e.g. horizontal, shear or distraction forces (e.g. seat-belt injury). In particular the importance of distraction has been neglected, for instance, Forsyth's valuable paper on cervical spine injuries does not differentiate properly between a distraction force under the chin (backward rotation and distraction) and a blow on the forehead (backward rotation and compression) yet the resultant lesions show marked differences and require different managements.

Yet by applying the principles of elementary dynamics it is possible to classify spinal injuries in a simple unambiguous fashion which reveals both the mechanism 
of injury and the resulting anatomical lesion and which will usually indicate the correct approach to restoring alignment and stability and will at least indicate undesirable and unnecessary therapeutic measures. Figure I indicates some of the many external forces which cause spinal injuries.

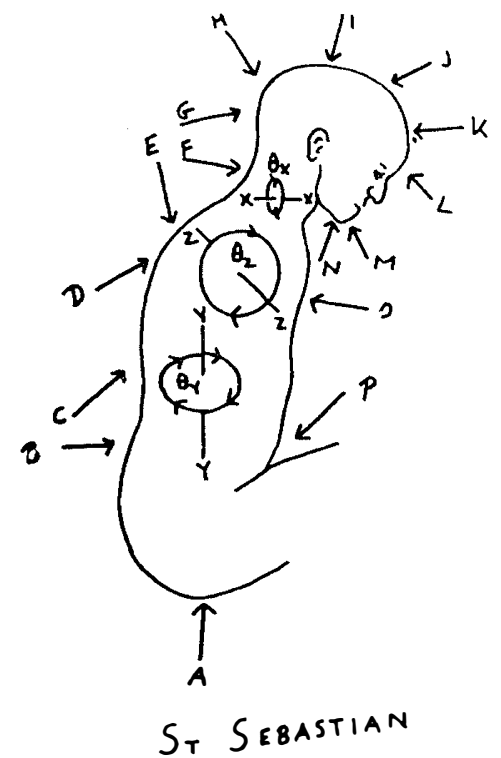

FIG. I
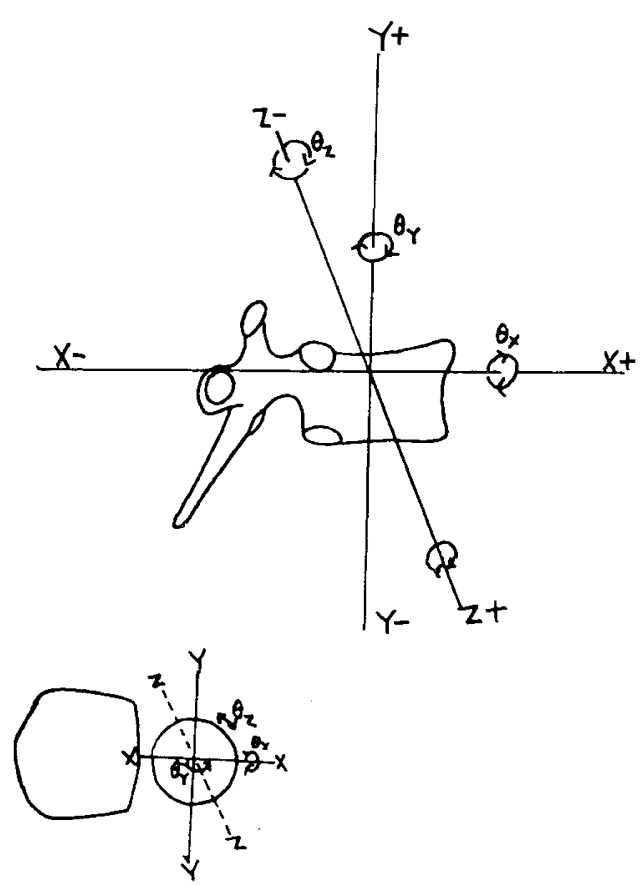

FIG. 2

Fig. I. - Example of a few of the many external forces which may impinge on the trunk and head and lead to spinal injuries. It will be obvious that opposing arrows may have similar action, e.g. a compression force at $I$ has many similarities with the compression force at $A$ and distraction force at $C$ may have the same effect as a distraction force at $P$.

Fig. 2.-The possible direction of forces in three dimensional space $Y$ is the vertical axis, $X$ and $Z$ are horizontal axes, $X$ being sagittal and $Z$ being coronal. Angular forces are represented round the axis of rotation in which they act. The small inset diagram illustrates how the forces acting on the human body are similar to those imparted by a golf club to a golf ball, both as regards direction and rotation.

The summative action of a number of forces acting on a rigid body can be fully described:

I. By indicating the direction of the resultant vector force in relation to the three dimensional coordinates. Mathematically these are labelled $x, y$ and $z$. Anatomically these are usually termed sagittal, cephalo-caudal and frontal. $y$ is the vertical axis, $x$ and $z$ horizontal axes.

2. In addition, if the forces are not equal and/or are not directed at the centre of gravity of the body they will impart a rotatory motion or (couple of forces) and again the axis of rotation can be described in relation to the three above men- 
tioned coordinates. Sagittal rotations are described as $\theta z^{\circ}$, horizontal rotations as $\theta y^{\circ}$, lateral or frontal rotations as $\theta x^{\circ}$, i.e. the supplementary letter indicates the axis of rotation (fig. 2) ( $\theta=$ theta).

Any combination of forces acting on a given vertebra will displace the vertebra according to the well-known principles of dynamics and the resultant displacement and accompanying tearing of ligaments, dislocations of joints, fractures of bones, etc., can be deduced with mathematical precision from the direction of the force.

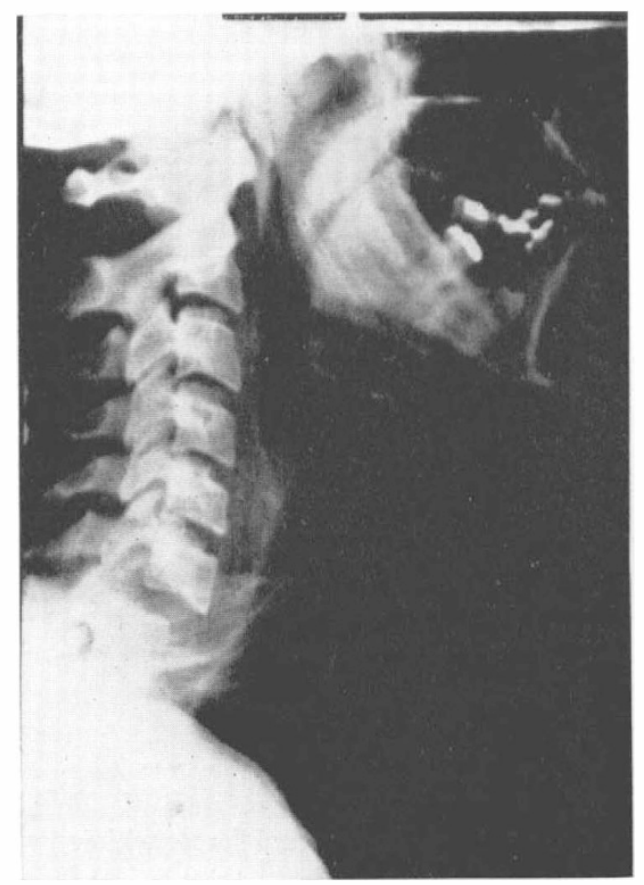

FIG. 3

Bilateral facet dislocation without cord damage due to a distraction and forward displacement force.

All spinal deformities following injury (indeed all spinal deformities) can, therefore, be best described in terms of the summation of the forces which produced the injury. These forces in their turn can be completely described in terms of their displacing and rotating actions, e.g. Figure 3 bilateral cervical dislocation without tetraplegia due to distraction and forward displacement.

There are great advantages in expressing the displacing forces in simple mathematical forms which can be universally understood. Therefore, displacements in the sagittal plane can be called $x+$ if forward, $x-$ if backward. Displacements in the cephalo-caudal plane can be called $y+$, if cephalic $y$ - if caudal; lateral displacements are described as arbitrarily $z+$ to the right, $z-$ to the left.

Forward rotation is called $\theta z+$, backward rotation $\theta z-$. Rotation movements in the horizontal plane are described by $\theta y$. Lateral flexion movements as $\theta x$. 
The following examples show how a number of fractures and dislocations of the spine can be described simply and comprehensively in this way (figs. 4 and 5):

I. Forces acting on spine to produce injuries.

$2,3,4,5$. Forces acting on individual vertebra.
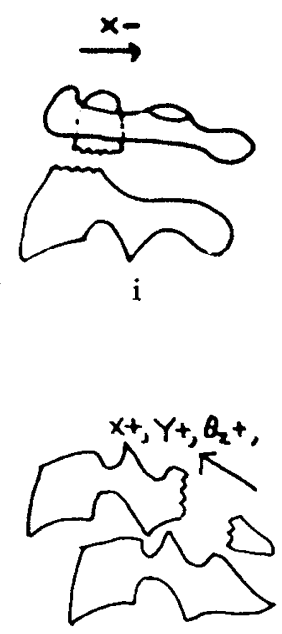

iv

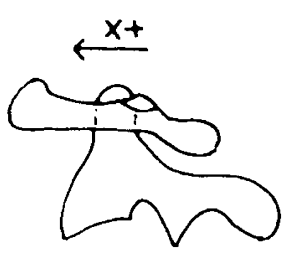

ii

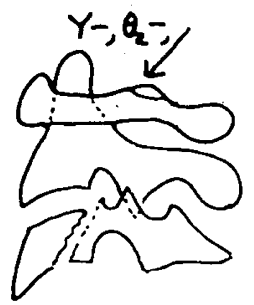

$\mathrm{v}$

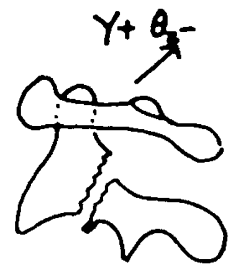

iii

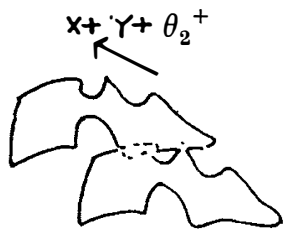

vi

FIG. 4

Examples of different types of fractures and dislocations of the spine with the direction on main causative force.

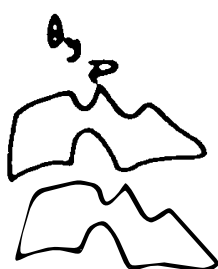

vii

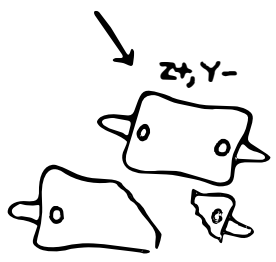

$\mathbf{x}$

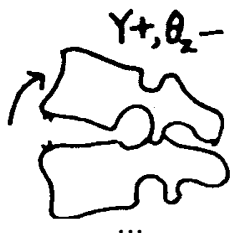

viii

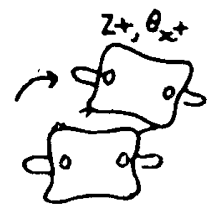

xi

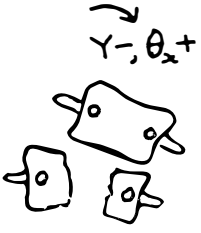

ix

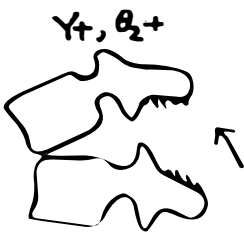

xii

FIG. 5

Examples of fractures and dislocations in the thoraco-lumbar and lumbar region with the main forces indicated.

i. Fractured odontoid-backward displacement injury $x-$.

ii. Atlanto axial dislocation-forward displacement injury $x+$. 


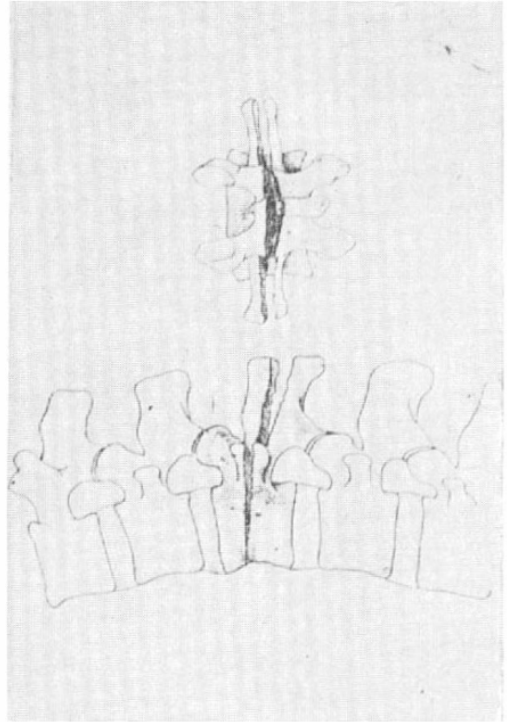

FIG. 6

Fig. 6.-Split fracture (Chance's fracture caused by distraction.

Fig. 7.-(after Smith \& Kaufer) mechanism of the distraction injury secondary to distraction injuries.

Fig. 8.-(after Smith \& Kaufer) further stage of the seat belt injury.

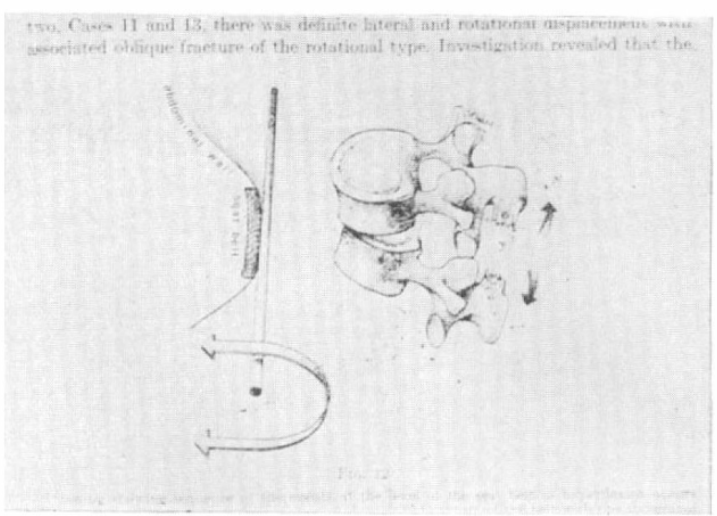

FIG. 7

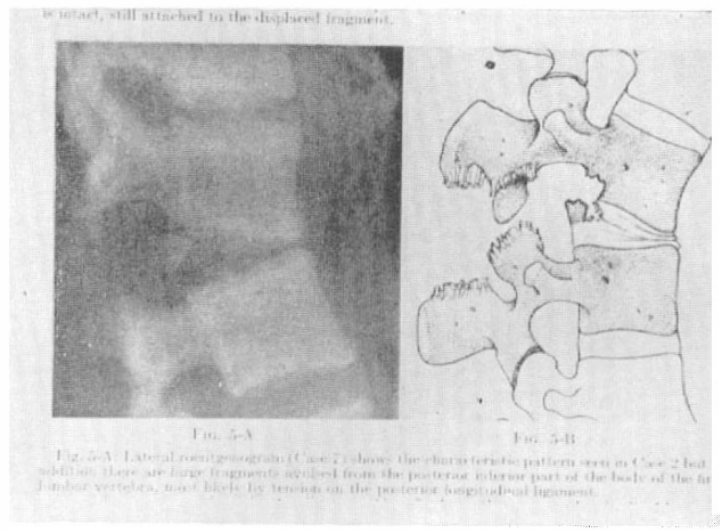

FIG. 8

iii. Fracture of the axis (ideal hangman's injury) cephalic and backward displacement (backward rotation) $y+, \theta z-$.

iv. TI fracture cephalic with forward rotation in the sagittal plane $x+, y+$, $\theta z+$

v. Vertical compression and backward rotation $y-, \theta z-$.

vi. Bilateral dislocation with forward displacement and upward displacement (cephalo-sagittal displacement) and forward rotation $x+, y+, \theta z+$.

vii. Unilateral dislocation and horizontal rotation injuries $\theta y$.

viii. Pure hyperextension $y+, \theta z-$.

ix. Crush fractures-vertical caudal force $(y-)$ with lateral displacement $\theta x$.

x. Thoraco-lumbar fracture with lateral displacement horizontal and lateral flexion $z+, y-$.

xi. Lateral bending-lateral rotation in the frontal plane $z+, \theta x+$.

xii. Upward displacement and rotation $y+, \theta z+$.

It is thus possible to describe all fractures and dislocations of the spine in terms of the direction of the force which produced the injury. The resulting 


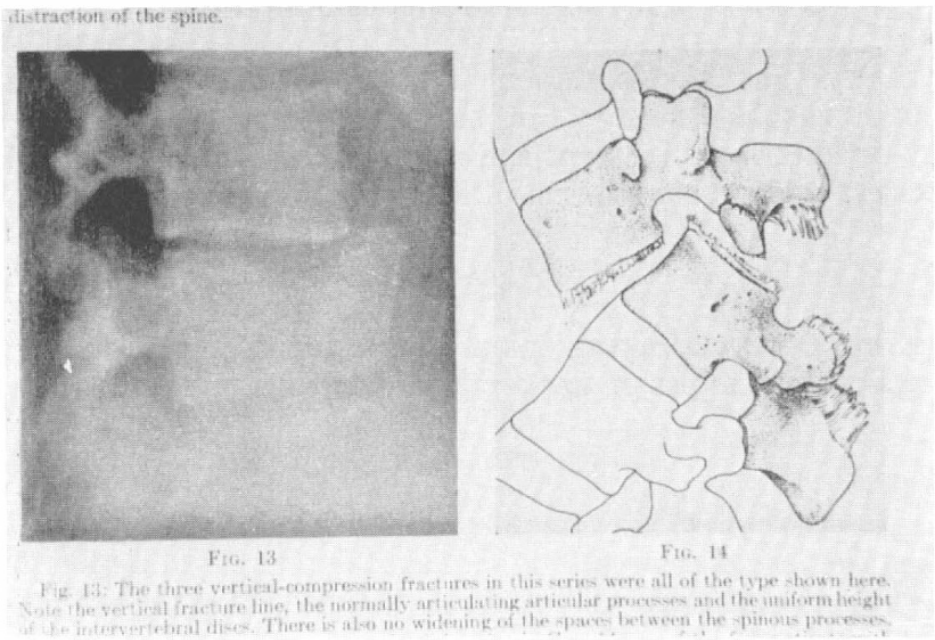

FIG. 9

(after Smith \& Kaufer) complete ligament disruption secondary to distraction injuries.

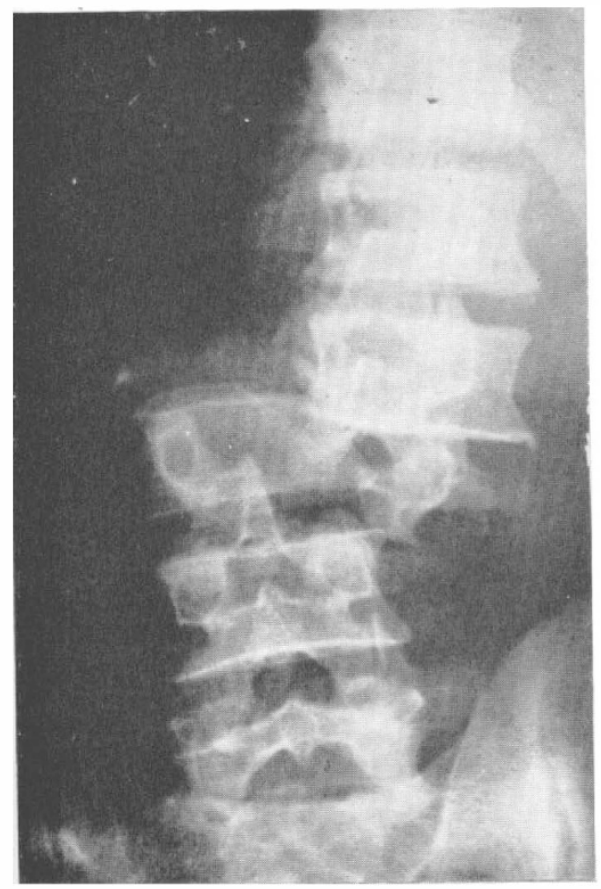

FIG. IO

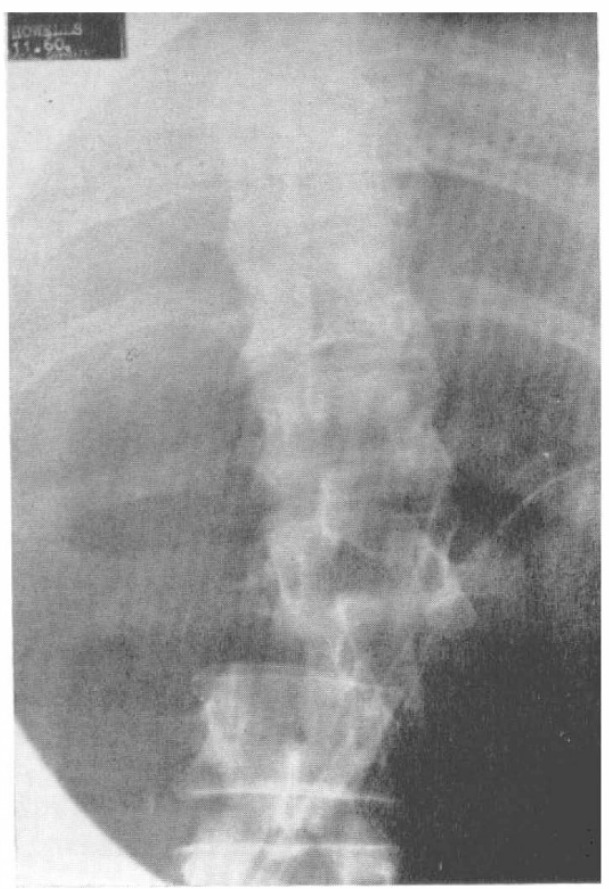

FIG. II

Fig. I0.-Forced lateral flexion injury. The line of the fracture running through the base of the pedicle. This injury was associated with minimal neurological involvement and following closed reduction the patient made a full recovery.

Fig. II.-Severe lateral flexion injury without bony damage producing complete and irreversible paraplegia. 
orthopaedic injury (fracture dislocation or torn ligament) can then be deduced. This will usually enable the surgeon to select the simplest, safest and most conservative way of restoring alignment and stability. It will at least enable him to avoid unnecessary and probably harmful operative interference.

\section{SUMMARY}

A plea is made for clasisfying spinal injuries according to the principles of classical dynamics. Examples of this are given and the advantages indicated. 